\title{
AXIOMATIC SHAPE THEORY
}

\section{PHILIP BACON}

ABSTRACT. The notion of shape theory is so defined that, if $\mathbf{H}$ is a category and $W$ is a subcategory of $H$, all shape theories on $(H, W)$ are isomorphic and, under a mild condition, a shape theory on $(H, W)$ always exists. Additional theorems facilitate the comparison of shape theories constructed by various means.

1. The recent proliferation of shape theories ([1], [3], [5], [7], [8], [9], [11]) suggests the desirability of a unified treatment of their common features. This note is primarily a study of aspects of shape theory that can be dealt with in terms of category theory; many of our definitions and theorems contain no topology.

If $\mathbf{A}$ is a category, $X \in A$ will mean that $X$ is an object of $\mathbf{A}$ and $f \epsilon$ $\mathbf{A}(X, Y)$ will mean that $f$ is a morphism of $\mathbf{A}$ with domain $X$ and codomain $Y$. If $X \in \mathbf{A}, \mathbf{A} X$ will denote the identity morphism in $\mathbf{A}(X, X)$. If $\mathbf{B}$ is a subcategory of $\mathbf{A}$ and $X \in \mathbf{A}$, let $\mathbf{A}(X, \mathbf{B})$ denote the class of morphisms of A with domain $X$ and codomain an object of $\mathbf{B}: \mathbf{A}(X, \mathbf{B})=\bigcup\{\mathbf{A}(X, Q) \mid Q \epsilon$ B . Throughout $\$ \$ 2-4$, let $\mathbf{H}$ be a category and $\mathbf{W}$ a subcategory of $\mathbf{H}$. For example, $\boldsymbol{H}$ could be the category of topological spaces and homotopy classes and $\mathbf{W}$ could be the category of polyhedra and homotopy classes.

2. This section deals with the definition, existence and uniqueness of shape theories. Suppose $\mathbf{G}$ is a category and $T: H \rightarrow G$ is a functor. A function $v: \mathbf{H}(X, W) \rightarrow \mathbf{G}(D, \mathbf{G})$ is said to be linked by $T$ if the following conditions hold:

(L1) If $Q \in \mathbb{W}$ and $k \in \mathbf{H}(X, Q)$, then $v k \in \mathbf{G}(D, T Q)$.

(L2) If $r \in \mathbb{W}(Q, P)$ and $k \in \mathbf{H}(X, Q)$, then $v(r k)=(T r)(v k)$.

(If $\mathbf{H}(X, \mathbb{W})$ and $\mathbf{G}(D, \mathbf{G})$ are endowed with the structure of a comma category $[4, \mathrm{p} .28]$, then $v$ is a functor.) If $X \in \mathbf{H}$, we say $T$ is $\mathbf{W}$-continuous at $X$ if, given any $D \in \mathbf{G}$ and any function $v: \mathbf{H}(X, \mathbb{W}) \rightarrow \mathbf{G}(D, \mathbf{G})$ linked by $T$, there is a unique $g \in \mathbf{G}(D, T X)$ such that $(T K) g=v k$ whenever $k \epsilon$ $\mathbf{H}(X, \mathbb{W}), T$ is $\mathbb{W}$-continuous if it is $\mathbf{W}$-continuous at each object $\mathbf{X}$ of $\mathbf{H}$.

A pair $(\mathbf{C}, C)$ is a shape theory on $(\mathbf{H}, \mathbb{W})$ if $\mathbf{C}$ is a category and $C$ : $\mathbf{H} \rightarrow \mathbf{C}$ is a functor satisfying the following conditions:

(S1) The objects of $\mathbf{C}$ are the objects of $\mathbf{H}$; if $X \in \mathbf{H}, C X=X$.

Presented to the Society, August 16, 1974; received by the editors June 21, 1974 and, in revised form, October 14, 1974.

AMS (MOS) subject classifications (1970). Primary 55D99; Secondary 18A30. 
(S2) If $Q \in \mathbb{W}$ and $s \in \mathbf{C}(X, Q)$, then there is just one $k \in \mathbf{I I}(X, Q)$ such that $C k=s$.

(S3) $C$ is Wocontinuous.

A similar definition has been given by Holsztyński [5].

Suppose $(\mathbf{H}, \mathbb{W})$ has the property that, if $X \in \mathbf{H}$, there is a set $J$ of objects of $W$ such that any morphism from $X$ to an object of $W$ factors through an object in $J$. Then there is a shape theory on (II, W). To see this, define a category $\mathbf{S}$ and a functor $S: H \rightarrow S$ by the method of Holsztyński [5, p. $161, S$ ] (see also [7] and [8]). The objects of $S$ are the objects of $H:$ if $X \in \mathbf{H}, S X=X . \mathbf{S}(X, Y)$ is the class of functions $v: \mathbf{H}(Y, \mathbb{W}) \rightarrow \mathbf{H}(X, \mathbf{H})$ linked by the identity functor on $\mathbf{H}$. (The hypothesis on $(\mathbf{H}, \mathbb{W})$ insures that the class $\mathbf{S}(X, Y)$ is a set.) If $v \in \mathbf{S}(X, Y)$ and $u \in \mathbf{S}(Y, Z)$, the composition $u v \in \mathbf{S}(X, X)$ is defined by $(u v) k=v(u k)$ whenever $k \in \mathbf{H}(Z, W)$. If $f \in$ $\mathbf{H}(X, Y),(S f) k=k f$ whenever $k \in \mathbf{H}(Y, W)$.

Theorem 2.1. $(\mathbf{S}, S)$ is a shape theory on $(\mathbf{H}, \mathbf{W})$.

Proof. To verify (S2) suppose $Q \in \mathbb{W}$ and $b \in \mathbf{S}(Z, Q)$. If $r \in \mathbf{H}(Q, \mathbb{W})$, then $(S(b(\mathbf{H} Q))) r=r(b(\mathbf{H} Q))=b r$. Hence

$$
S(b(I I Q))=b .
$$

To see that $b(\mathbf{H} Q)$ is unique, suppose $c \in \mathbf{H}(X, Q)$ and $S c=b$. Then $c=$ $(\mathbf{H} Q)_{c}=\left(S_{C}\right)(\mathbf{H} Q)=b(\mathbf{H} Q)$.

To verify (S3), suppose $v: \mathbf{H}(X, \mathbb{W}) \rightarrow \mathbf{S}(Z, \mathbf{S})$ is a function linked by $S$. If $k \in \mathbf{H}(X, \mathbf{W})$, by (S2) there is a unique $g k \in \mathbf{I}(Z, \mathbb{W})$ such that $S(g k)=v k$. This defines a function $g: \mathbf{H}(X, W) \rightarrow \mathbf{H}(X, \mathbf{H})$ that satisfies (L1). Suppose $r \in \mathbb{W}(Q, P)$ and $k \in \mathbf{H}(X, Q)$. Then

$$
S(g(r k))=v(r k)==(S r)(v k)=(S r)(S(g k))=S(r(g k)) .
$$

By (S2), $g(r k)=r(g k)$. Thus $g$ satisfies (L2) as well as (L1), that is, $g \in$ $\mathrm{S}(Z, X)$.

Next we show that $(S k) g=v k$ whenever $k \in \mathbf{H}(X, \mathbb{W})$. Suppose $Q \in \mathbb{W}$ and $k \in \mathbf{H}(X, Q)$. If $P \in \mathbb{W}$ and $r \in \mathbf{H}(Q, P)$, then

$$
\begin{aligned}
S(((S k) g) r) & =S(g((S k) r)) & & \text { by the definition of composition in } \mathbf{S}, \\
& =S(g(r k)) & & \text { by the definition of } S, \\
& =v(r k) & & \text { by the definition of } g, \\
& =(S r)(v k) & & \text { since } v \text { is linked by } S, \\
& =(S r)(S((v k)(\mathbf{H} Q))) & & \text { by }(*), \\
& =S(r((v k)(\mathbf{H} Q))) & & \text { since } S \text { is a functor, } \\
& =S((v k)(r(\mathbf{H} Q))) & & \text { since } v k \text { is linked by the identity on } \mathbf{H}, \\
& =S\left((v k)_{r}\right) . & &
\end{aligned}
$$

By $(\mathrm{S} 2),((S k) g) r=(v k) r$. Hence $(S k) g=v k$. 
Suppose $f \in \mathbf{S}(Z, X)$ and $(S k) f=v k$ whenever $k \in \mathbf{H}(X, W)$. If $r \in \mathbb{W}(Q, P)$ and $k \in \mathbf{H}(X, Q)$, then

$$
(S(f k)) r=r(f k)=f(r k)=f((S k) r)=((S k) f) r .
$$

So $S(f k)=(S k) f=v k=S(g k)$. By (S2), $f k=g k$. Hence $f=g$.

Theorem 2.2. Suppose $\mathbf{C}$ is a category and $\mathrm{C}: \mathbf{H} \rightarrow \mathbf{C}$ is a functor satisfying (S1) and (S2). If $\mathbf{G}$ is a category and $T: \mathbf{H} \rightarrow \mathbf{G}$ is a $\mathbf{W}$-continuous functor, then there is just one functor $R: \mathbf{C} \rightarrow \mathbf{G}$ such that $R C=T$.

Proof. If $X \in \mathbf{C}$, define $R X=T X$. Suppose $f \in \mathbf{C}(X, Y)$. If $Q \in \mathbb{W}$ and $k \in \mathbf{H}(Y, Q)$, let $u_{f} k \in \mathbf{H}(X, Q)$ be the unique morphism such that $C\left(u_{f} k\right)=$ $(C k) f$. This defines a function $u_{f}: \mathbf{H}(Y, W) \rightarrow \mathbf{H}(X, \mathbf{H})$ satisfying (L1). If $r \in \mathbb{W}(Q, P)$ and $k \in \mathbf{H}(Y, Q)$, then

$$
C\left(r\left(u_{f} k\right)\right)=(C r)\left(C\left(u_{f} k\right)\right)=(C r)(C k) f=(C(r k)) f=C\left(u_{f}(r k)\right) .
$$

By (S2), $r\left(u_{f} k\right)=u_{f}(r k)$, that is, $u_{f}$ satisfies (L2). Define $v_{f}: \mathbf{H}(Y, W) \rightarrow$ $\mathbf{G}(T X, \mathbf{G})$ by $v_{f} k=T\left(u_{f} k\right)$. Since $u_{f}$ is linked by the identity, $v_{f}$ is linked by $T$. Since $T$ is $\mathbb{W}$-continuous at $T$, there is a unique $R f \in \mathbf{G}(T X, T Y)$ such that $(T k)(R f)=v_{f} k$ whenever $k \in \mathbf{H}(Y, \mathbb{W})$.

If $Y=X$ and $f=\mathbf{C} X$, then $k=u_{f} k$ and $(T k)(\mathbf{G}(R X))=T k=v_{f} k=(T k)(R f)$ whenever $k \in \mathbf{H}(X, W)$. Since $T$ is $\mathbf{W}$-continuous at $X, \mathbf{G}(R X)=R f$.

Suppose $f \in \mathbf{C}(X, Y)$ and $g \in \mathbf{C}(Y, Z)$. If $k \in \mathbf{H}(Z, W)$, then

$$
C\left(u_{g f} k\right)=(C k) g f=\left(C\left(u_{g} k\right)\right) f=C\left(u_{f}\left(u_{g} k\right)\right) .
$$

By (S2), $u_{g f} k=u_{f}\left(u_{g} k\right)$. Using this we compute

$$
\begin{aligned}
(T k)(R(g f)) & =v_{g f} k=T\left(u_{g f} k\right)=T\left(u_{f}\left(u_{g} k\right)\right)=v_{f}\left(u_{g} k\right) \\
& =\left(T\left(u_{g} k\right)\right)(R f)=\left(v_{g} k\right)(R f)=(T k)(R g)(R f) .
\end{aligned}
$$

Since $T$ is $\mathbb{W}$-continuous at $Z, R(g f)=(R g)(R f)$. Thus $R$ is a functor.

If $f \in \mathbf{H}(X, Y)$ and $k \in \mathbf{H}(Y, W)$, then $C\left(u_{C f} k\right)=(C k)(C f)=C(k f)$. By (S2), $u_{C f} k=k f$. Hence

$$
(T k)(R(C f))=v_{C f} k=T\left(u_{C f} k\right)=T(k f)=(T k)(T f) .
$$

Since $T$ is $\mathbb{W}$-continuous at $Y, R(C f)=T f$. Thus $R C=T$.

Suppose $R^{\prime}: \mathbf{C} \rightarrow \mathbf{G}$ is a functor such that $R^{\prime} C=T$. If $f \in \mathbf{C}(X, Y)$ and $k \in \mathbf{I I}(Y, \mathbb{W})$, then

$$
(T k)\left(R^{\prime} f\right)=\left(\left(R^{\prime} C\right) k\right)\left(R^{\prime} f\right)=R^{\prime}((C k) f)=R^{\prime}\left(C\left(u_{f} k\right)\right):=T\left(u_{f} k\right)=v_{f} k=(T k)(R f) .
$$

Since $T$ is $W$-continuous at $Y, R^{\prime} f=R f$. Thus $R^{\prime}=R$. This completes the proof of Theorem 2.2.

A functor $R: \mathbf{C} \rightarrow \mathbf{I}$ is called an isomorphism if there is a functor 
$P:$ I $\rightarrow \mathrm{C}$ such that $R P$ and $P R$ are identity functors. A fourfold application of Theorem 2.2 proves

Theorem 2.3. If each of (C, C) and (I), D) is a shape theory on (H, W), then there is a unique isomorphism $R: \mathbf{C} \rightarrow \mathbf{D}$ such that $R C=D$.

3. Throughout this section assume that $\mathbf{N}$ is a subcategory of W. We shall develop conditions under which a pair $(\mathbf{C}, C)$ is a shape theory on both $(\mathbf{H}, \mathbb{W})$ and $(\mathbf{H}, \mathbf{N})$.

Lemma 3.1. If $T: \mathbf{H} \rightarrow \mathbf{G}$ is an $\mathbf{N}$-continuous functor, then $T$ is $\mathbf{W}$-continuous.

I'roof. Suppose $v: \mathbf{H}(X, \mathbb{W}) \rightarrow \mathbf{G}(D, \mathbf{G})$ is linked by $T$. Let $u: \mathbf{H}(X, \mathbf{N})$ $\rightarrow \mathbf{G}(D, \mathbf{G})$ be the restriction of $v ; u$ is linked by $T$. Since $T$ is $\mathbf{N}$-continuous at $X$, there is a $g \in \mathbf{G}(D, T X)$ such that, whenever $k \in \mathbf{H}(X, \mathbf{N}),(T k) g$ $=u k$.

Suppose $Q \in \mathbb{W}$ and $k \in \mathbf{H}(X, Q)$. If $r \in \mathbf{H}(Q, \mathbf{N})$, then

$$
(T r)(T k)_{g}=(T(r k))_{g}=u(r k)=v(r k)=(T r)(v k) \text {. }
$$

Since $T$ is $\mathbf{N}$-continuous at $Q,(T k) g=v k$.

Suppose $f \in \mathbf{G}(D, T X)$ and $(T k) f=v k$ whenever $k \in \mathbf{H}(X, \mathbb{W})$. Then, in particular, $(T k) f=u k$ whenever $k \in \mathbf{H}(X, \mathbf{N})$. Since $T$ is $\mathbf{N}$-continuous at $X$, $f=g$. Thus $T$ is $\mathbb{H}$-continuous and Lemma 3.1 is proved.

An object $Y$ of $\mathbf{H}$ is said to dominate an object $X$ of $\mathbf{H}$ if there exist morphisms $i \in \mathbf{H}(X, Y)$ and $j \in \mathbf{H}(Y, X)$ such that $j i=\mathbf{H} X$.

Lemma 3.2. If $\mathbf{N}$ is full in $\mathbf{H}$, if every object of $\mathbb{W}$ is dominated by an object of $\mathbf{N}$, if $X \in \mathbf{H}$, and if $T: \mathbf{H} \rightarrow \mathbf{G}$ is a functor $\mathbf{H}$-continuous at $X$, then $T$ is N-continuous at $X$.

Proof. Suppose $v: \mathbf{H}(X, \mathbf{N}) \rightarrow \mathbf{G}(D, \mathbf{G})$ is linked by $T$. If $Q \in \mathbb{W}$, let $M_{Q} \in \mathbf{N}, i_{Q} \in \mathbf{H}\left(Q, M_{Q}\right)$ and $j_{Q} \in \mathbf{H}\left(M_{Q}, Q\right)$ be such that $j_{Q} i_{Q}=\mathbf{H} Q$. In particular, if $Q \in \mathbf{N}$, choose $M_{Q}=Q$ and $i_{Q}=j_{Q}=\mathbf{H} Q$. If $k \in \mathbf{H}(X, Q)$, define $u k=\left(T j_{Q}\right)\left(v\left(i_{Q} k\right)\right)$. If $r \in \mathbb{W}(Q, P)$ and $k \in \mathbf{H}(X, Q)$, then

$$
\begin{aligned}
u(r k) & \left.=\left(T j_{P}\right)\left(v_{P} i_{P} r\right)\right)=\left(T j_{P}\right)\left(v\left(i_{P} r j_{Q} i_{Q} k\right)\right)=\left(T j_{P}\right)\left(T\left(i_{P} r j_{Q}\right)\right)\left(v\left(i_{Q} k\right)\right) \\
& =(T r)\left(T j_{Q}\right)\left(v\left(i_{Q} k\right)\right)=(T r)(u k) .
\end{aligned}
$$

Thus the function $u: \mathbf{H}(X, \mathbb{W}) \rightarrow \mathbf{G}(D, \mathbf{G})$ is linked by $T$. Since $T$ is $\mathbb{W}$-continuous at $X$, there is a unique $g \in \mathbf{G}(D, T X)$ such that $(T k) g=u k$ whenever $k \in \mathbf{I}(X, W)$. Since $u$ is an extension of $v,(T k) g=v k$ whenever $k \in \mathbf{H}(X, \mathbf{N})$. Suppose $f \in \mathbf{G}(D, T X)$ and $(T k) f=v k$ whenever $x \in \mathbf{H}(X, \mathbf{N})$. If $Q \in \mathbb{W}$ and $k \in \mathbf{I}(X, Q)$, then $(T k) f=\left(T j_{Q}\right)\left(T\left(i_{Q} k\right)\right) f=\left(T j_{Q}\right)\left(v\left(i_{Q} k\right)\right)=u k$. Since $T$ is $\mathbb{W}$-continuous at $X, f=g$. 
Theorem 3.3. Suppose $\mathbf{N}$ is full in $\mathbf{W}$, each object of $\mathbb{W}$ is dominated by an object of $\mathbf{N}$, and $C: \mathbf{H} \rightarrow \mathbf{C}$ is a functor. Then $(\mathbf{C}, C)$ is a shape theory on $(\mathbf{H}, \mathbf{W})$ if and only if $(\mathrm{C}, C)$ is a shape theory on $(\mathbf{H}, \mathbf{N})$.

Proof. Suppose $(\mathrm{C}, C)$ is a shape theory on $(\mathbf{H}, \mathbf{N})$. By Lemma 3.1, $C$ is $\mathbb{W}$-continuous, Suppose $Q \in \mathbb{W}$ and $s \in \mathbf{C}(X, Q)$. Let $M \in \mathbf{N}, i \in \mathbf{H}(Q, M)$ and $j \in \mathbf{H}(M, Q)$ be such that $j i=\mathbf{H} Q$. There is a unique $f \in \mathbf{H}(X, M)$ such that $C f=(C i) s$. Then $C(j f)=(C j)(C f)=(C j)(C i) s=s$. Suppose $g \in \mathbf{H}(X, Q)$ is such that $C g=s$. Then $C(i g)=(C i)(C g)=(C i) s=C f$. Since $(\mathrm{C}, C)$ satisfies (S2) as a shape theory on $(\mathbf{H}, \mathbf{N}), i g=f$. Consequently $g=j i g=j f$. Thus $(\mathbf{C}, C)$ satisfies $(\mathrm{S} 2)$ as a shape theory on $(\mathbf{H}, \mathbf{W})$.

By Lemma 3.2 any shape theory on $(\mathbf{H}, \mathbf{W})$ is a shape theory on $(\mathbf{H}, \mathbf{N})$.

4. In this section we suppose that $\mathbf{G}$ is a category, $T: \mathbf{H} \rightarrow \mathbf{G}$ is a functor, $X \in \mathbf{H}, A$ is a directed set, and $\left\{X_{i} \in \mathbf{H} \mid i \in A\right\},\left\{p_{i j} \in \mathbf{H}\left(X_{j}, X_{i}\right) \mid i, j \in\right.$ $A, j>i\},\left\{p_{i} \in \mathbf{H}\left(X, X_{i}\right) \mid i \in A\right\}$ are such that the following conditions hold:

(B1) $\left(\left\{X_{i}\right\}_{9}\left\{p_{i j}\right\}\right)$ is an inverse system.

(B2) $p_{i j} p_{j}=p_{i}$ whenever $i, j \in A$ and $j>i$.

(B3) If $Q \in \mathbb{W}$ and $k \in \mathbf{H}(X, Q)$, then there is an $i \in A$ and a $b \in \mathbf{H}\left(X_{i}, Q\right)$ such that $k=b p_{i}$.

Theorem 4.1. If each $X_{i}$ is in $\mathbb{W}$, if each $p_{i j}$ is in $\mathbb{W}\left(X_{j}, X_{i}\right)$ and if $\left(T X,\left\{T p_{i}\right\}\right)$ is an inverse limit of $\left(\left\{T X_{i}\right\},\left\{T p_{i j}\right\}\right)$, then $T$ is W-continuous at $X$.

Proof. Suppose $D \in \mathbf{G}$ and $v: \mathbf{H}(X, \mathbb{W}) \rightarrow \mathbf{G}(D, \mathbf{G})$ is linked by $T$. For each $i \in A$, let $g_{i}=v p_{i}$. If $j>i, p_{i j} g_{j}=p_{i j}\left(v p_{i}\right)=v\left(p_{i j} p_{j}\right)=v p_{i}=g_{i}$. By the definition of inverse limit, there is a unique $g \in \mathbf{G}(D, T X)$ such that $\left(T p_{i}\right) g=g_{i}$ whenever $i \in A$. Suppose $Q \in \mathbb{W}$ and $k \in \mathbf{H}(X, Q)$. By (B3) there is an $i \in A$ and a $b \in \mathbf{H}\left(X_{i}, Q\right)$ such that $k=b p_{i}$. Hence

$$
(T k) g=(T b)\left(T p_{i}\right) g=(T b)\left(v p_{i}\right)=v\left(b p_{i}\right)=v k .
$$

Suppose $f \in \mathbf{G}(D, T X)$ and $(T K) f=v k$ whenever $k \in \mathbf{H}(X, W)$. Then, in particular, $\left(T p_{i}\right) f=g_{i}$ whenever $i \in A$. By the definition of inverse limit $f=g$.

Theorem 4.2. Suppose that, whenever $Q \in \mathbb{W}, i \in A, k_{t} \in \mathbf{H}\left(X_{i}, Q\right)(t=$ $1,2)$, and $k_{1} p_{i}=k_{2} p_{i}$, there is a $j>i$ such that $k_{1} p_{i j}=k_{2} p_{i j}$. If $T$ is W-continuous, then $\left(T X,\left\{T p_{i}\right\}\right)$ is an inverse limit of $\left(\left\{T X_{i}\right\},\left\{T p_{i j}\right\}\right)$.

Proof. Suppose $\left\{g_{i} \in \mathbf{G}\left(D, T X_{i}\right) \mid i \in A\right\}$ is such that $\left(T p_{i j}\right) g_{j}=g_{i}$ whenever $i, j \in A$ and $j>i$. We must show that there is just one $g \in \mathbf{G}(D, T X)$ such that $\left(T p_{i}\right) g=g_{i}$ whenever $i \in A$.

If $Q \in \mathbb{W}$ and $k \in \mathbf{H}(X, Q)$, then, by (B3), there is an $m(k) \in A$ and a $b_{k} \in \mathbf{H}\left(X_{m(k)}, Q\right)$ such that $k=b_{k} p_{m(k)}$. Define $v k=\left(T b_{k}\right) g_{m(k)}$. Suppose $r \in \mathbb{W}(Q, P)$ and $k \in \mathbf{H}(X, Q)$. Let $i>m(k), m(r k)$. Then 


$$
r b_{k} p_{m(k) i} p_{i}=r b_{k} p_{m(k)}=r k=b_{r k} p_{m(r k)}=b_{r k} p_{m(r k) i} p_{i}
$$

By hypothesis there is a $j>i$ such that $r b_{k} p_{m(k) i} p_{i j}=b_{r k} p_{m(r k) i} p_{i j}$, which simplifies to $r b_{k} p_{m(k) j}=b_{r k} p_{m(r k) j}$. Using this we compute

$$
\begin{aligned}
(T r)(v k) & =(T r)\left(T b_{k}\right) g_{m(k)}=(T r)\left(T b_{k}\right)\left(T p_{m(k) j}\right) g_{j} \\
& =\left(T b_{r k}\right)\left(T p_{m(r k) j}\right) g_{j}=\left(T b_{r k}\right) g_{m(r k)}=v(r k) .
\end{aligned}
$$

Thus $v: \mathbf{H}(X, \mathbb{W}) \rightarrow \mathbf{G}(D, \mathbf{G})$ is linked by $T$. Since $T$ is $\mathbb{W}^{\circ}$-continuous at $X$, there is a unique $g$ in $\mathbf{G}(D, T X)$ such that $(T k) g=v k$ whenever $k \in \mathbf{H}(X, W)$.

Suppose $a \in A, Q \in \mathbb{W}$ and $k \in \mathbf{H}\left(X_{a}, Q\right)$. Define $e=k p_{a}$. Choose $i>$ $a, m(e)$. Then

$$
k p_{a i} p_{i}=k p_{a}=e=b_{e} p_{m(e)}=b_{e} p_{m(e) i} p_{i}
$$

By hypothesis there is a $j>i$ such that $k p_{a i} p_{i j}=b_{e} p_{m(e) i} p_{i j}$, which simplifies to $k p_{a j}=b_{e} p_{m(e) j}$. Using this we compute

$(T k)\left(T p_{a}\right) g=(T e) g=v e=\left(T b_{e}\right) g_{m(e)}=\left(b_{e}\right)\left(T p_{m(e) j}\right)_{o j}=(T k)\left(T p_{a j}\right) g_{j}=(k) g_{a}$.

Since $T$ is $\mathbb{W}$-continuous at $X_{a},\left(T p_{a}\right) g=g_{a}$.

Suppose $f \in \mathbf{G}(D, T X)$ and $\left(T p_{a}\right) f=g_{a}$ whenever $a \in A$. If $k \in \mathbf{H}(X, W)$, then

$$
(T k) f=\left(T b_{k}\right)\left(T p_{m(k)}\right) f=\left(T b_{k}\right) g_{m(k)}=v k .
$$

Since $T$ is $\mathbb{W}$-continuous at $X, f=g$.

5. This section contains an application of the theorems of $\$ 4$. Let $M$ be a full subcategory of the category of topological spaces and homotopy classes. If $X, Y \in \mathbf{M}$ and $X \subset Y$, let $i_{Y X} \in \mathbf{M}(X, Y)$ denote the homotopy class of the inclusion map and let $\operatorname{Nbd}(Y, X)$ denote the set of all $N \in M$ such that $X \subset$ Int $N$ and $N \subset Y$. A functor $T$ from $\mathbf{M}$ to an arbitrary category $\mathbf{G}$ is called weakly continuous if, given $X, Y \in \mathbf{M}$ with $X$ a closed subspace of $Y,\left(T X,\left\{T i_{N X} \mid N \in \operatorname{Nbd}(Y, X)\right\}\right)$ is an inverse limit of

$$
\left(\{T N \mid N \in \operatorname{Nbd}(Y, X)\},\left\{T i_{L N} \mid L, N \in \operatorname{Nbd}(Y, X), N \subset L\right\}\right) .
$$

Let $\mathbf{E}$ be the full subcategory of $\mathbf{M}$ whose objects are those spaces in $\mathbf{M}$ that are neighborhood extensors for $\mathbf{M}$. We suppose that $\mathbf{M}$ satisifes the following conditions:

(A1) If $X \in \mathbf{M}$, then $X \times I \in \mathbf{M}$ (where $I=[0,1]$ ).

(A2) If $X, Y \in \mathbf{M}$, if $X$ is a closed subset of $Y$, and if $U$ is a neighborhood of $X$, then there is a neighborhood $N$ of $X$ such that $N \subset U$ and $N \in \mathbf{M}$.

(A3) If $X \in \mathbf{M}$, there is a $Y \in \mathbf{M}$ such that $X$ is a closed subspace of $Y$ and, given any neighborhood $U$ of $X$, there is a neighborhood $N$ of $X$ such that $N \subset U$ and $N \in \mathbf{E}$. 
Lemma 5.1. Suppose $X, Y \in \mathbf{M}, X$ is a closed subspace of $Y, Q \in \mathbf{E}, L$ $\epsilon \operatorname{Nbd}(Y, X), h_{t} \in \mathbf{M}(L, Q)(t=0,1)$ and $h_{0} i_{L X}=h_{1}{ }^{i} L X$. Then there is an $N$ in $\operatorname{Nbd}(Y, X)$ such that $N \subset L$ and $h_{0}{ }_{L N}=h_{1} i_{L N}$.

Proof. Let $f_{t}: L \rightarrow Q$ be a map in the homotopy class $h_{t}(t=0,1)$. Since $h_{0} i_{L X}=h_{1}{ }^{i} L X$, there is a map $m: X \times I \rightarrow Q$ such that $m(x, t)=f_{t} x$ whenever $(x, t) \in X \times\{0,1\}$. Let $Z=X \times I \cup L \times\{0,1\} ; Z$ is a closed subspace of $L \times I$. Define a map $k: Z \rightarrow Q$ by $k(x, t)=m(x, t)$ when $(x, t) \epsilon$ $X \times I ; k(x, t)=f_{t} x$ when $(x, t) \in L \times\{0,1\}$. Since $Q \in \mathbf{E}$, there is a neighborhood $B$ of $Z$ and an extension of $k$ to a map $j: B \rightarrow Q$. There is a neighborhood $D$ of $X$ such that $D \times I \subset B$. By (A2) there is a neighborhood $N$ of $X$ such that $N \subset D$ and $N \in \mathrm{M}$. The restriction of $j$ to $N \times I$ is a homotopy from $f_{0} \mid N$ to $f_{1} \mid N$. So $b_{0} i_{L N}=h_{1} i_{L N}$.

Theorem 5.2. If $\mathbf{G}$ is a category and $T: \mathbf{M} \rightarrow \mathbf{G}$ is a functor, then the following are equivalent:

(1) $T$ is weakly continuous.

(2) $T$ is E-continuous.

Proof that (1) implies (2). Suppose $T$ is weakly continuous and $X \in \mathbf{M}$. By (A3), $X$ may be regarded as a closed subspace of a space $Y$ in $\mathbf{M}$ such that $\operatorname{Nbd}(Y, X)$ contains a cofinal subcollection $\left\{X_{i} \mid i \in A\right\}$ of spaces in $\mathbf{E}$. Since $T$ is weakly continuous, $T X$ is an inverse limit of $\left\{T X_{i} \mid i \in A\right\}$. By (A2) and the definition of neighborhood extensor (B3) holds. By Theorem 4.1, $T$ is E-continuous at $X$.

Proof that (2) implies (1). Suppose $T$ is E-continuous and $X, Y \in M$ with $X$ a closed subspace of $Y$. By Lemma 5.1 and Theorem 4.2, TX is an inverse limit of $\{T N \mid N \in \operatorname{Nbd}(Y, X)\}$.

Theorem 5.2 shows that weak continuity can be used instead of E-continuity in our axioms for a shape theory on (M, E). The category of metrizable spaces and homotopy classes furnishes an example of a category satisfying (A1)-(A3). (A3) holds because every metrizable space is embeddable as a closed set in a normed linear space [10], every normed linear space is an extensor for metrizable spaces [2, p. 188, Theorem 6.1], and open subsets of neighborhood extensors are neighborhood extensors [6, p. 42, Proposition 6.1].

\section{REFERENCES}

1. K. Borsuk, Concerning homotopy properties of compacta, Fund. Math. 62 (1968), 223-254. MR 37 \#4811.

2. J. Dugundji, Topology, Allyn and Bacon, Boston, Mass., 1966. MR 33 \#1824.

3. R. H. Fox, On shape, Fund. Math. 74 (1972), 47-71. MR 45 \#5973.

4. H. Herrlich and G. E. Strecker, Category theory: An introduction, Allyn and Bacon, Boston, Mass., 1973. 
5. W. Holsztyński, An exten sion and axiomatic characterization of Borsuk's theory of shape, Fund. Math. 70 (1971), 157-168. MR 43 \#8080.

6. S.-T. Hu, Theory of retracts, Wayne State Univ. Press, Detroit, Mich., 1965. MR $31 \# 6202$.

7. G. Kozlowski and J. Segal, On the shape of 0-dimensional paracompacta, Fund. Math. 83 (1974), 151-154.

8. S. Mardešić, Shapes for topological spaces, General Topology and Appl. 3 (1973), 265-282. MR $48 \# 2988$.

9. S. Mardešić and J. Segal, Shapes of compact and ANR-systems, Fund. Math. 72 (1971), 41-59. MR $45 \# 7686$.

10. E. Michael, A short proof of the Arens-Eells embedding theorem, Proc. Amer. Math. Soc. 15 (1964), 415-416. MR 28 \#5421.

11. T. Porter, Generalized shape theory, Proc. Roy. Irish Acad. Ser. A 74 (1974), $33-48$.

DEPARTMENT OF MATHEMATICS, UNIVERSITY OF FLORIDA, GAINESVILLE, FLORIDA 32611 